\title{
Artículo
}

\section{Envejecimiento y estrategias de adaptación a los entornos urbanos desde la gerontología ambiental}

\section{Aging and adaptation strategies to urban environments from environmental gerontology}

\author{
María Trinidad García-Valdez* \\ Diego Sánchez-González** \\ Rosario Román-Pérez***
}

\section{Resumen}

El estudio reflexiona sobre las estrategias de adaptación a los entornos urbanos en el envejecimiento, con alusiones a América Latina. Los resultados indican la existencia de problemas derivados del reduccionismo analítico y la simplificación de los modelos teóricos sobre la adaptación ambiental y el envejecimiento. La desadaptación está determinada por las capacidades personales (exclusión social, discapacidad y dependencia) y, principalmente, por los problemas fisico-sociales urbanos. Asimismo, se proponen estrategias de adaptación ambiental para el envejecimiento activo en el lugar, a partir de la evaluación de los activos personales, así como de los atributos y funciones del ambiente urbano.

Palabras clave: envejecimiento; adaptación ambiental; entornos urbanos; espacio público; exclusión social; gerontología ambiental.

* Centro de Investigación en Alimentación y Desarrollo, A.C. Dirección Postal: Carretera a la Victoria, Km. 0.6, 83304, Hermosillo, Sonora, México. Correo electrónico: maryt_1999@ yahoo.com

** Universidad Autónoma de Madrid. Dirección postal: Ciudad Universitaria de Cantoblanco, 28049, Madrid, España. Correo electrónico: diego.sanchezg@uam.es

*** Centro de Investigación en Alimentación y Desarrollo, A.C. Dirección postal: Carretera a la Victoria, Km. 0.6, 83304, Hermosillo, Sonora, México. Correo electrónico: rroman@ ciad.mx ORCID: http://orcid.org/0000-0002-2337-8917 


\begin{abstract}
The study reflects on the strategies of adaptation to the urban environments in aging, with allusions to Latin America. The results indicate the existence of problems derived from analytical reductionism and the simplification of the theoretical models on environmental adaptation and aging. Maladjustment is determined by personal abilities (social exclusion, disability and dependency) and, mainly, urban physicalsocial problems. Likewise, environmental adaptation strategies for active aging in the place are proposed from the evaluation of personal assets, as well as the attributes and functions of the urban environment.
\end{abstract}

Keywords: aging; environmental adaptation; urban environments; public space; social exclusión; environmental gerontology.

\title{
Introducción
}

El aumento del envejecimiento demográfico urbano se presenta como un desafío sin precedentes a nivel global, con especial incidencia en los países de las regiones en desarrollo (Rowles y Bernard, 2013). En el año 2050 una cuarta parte de la población mundial tendrá 60 y más años, y residirá principalmente en las grandes ciudades de las regiones en desarrollo como América Latina y el Caribe (ONU, 2015). Un ejemplo es México, con 12.4 millones de personas de 60 y más años, que representan $10.4 \%$ de la población total (INEGI, 2015), con una proyección de 14.08 millones en 2030 (Conapo, 2010).

La investigación indica que los genes y sobre todo el ambiente físico y social determinan los años de vida, aunque existe información limitada de sus conexiones con la calidad de vida en el envejecimiento. En las últimas cuatro décadas se ha reconocido la necesidad de construir conocimiento de carácter multidisciplinar que dé cuenta precisamente de cómo interactúan los entornos físico-sociales en el proceso de envejecimiento, lo que dio lugar al desarrollo de la gerontología ambiental. Ésta se nutre de disciplinas como la geografía humana, la psicología, la sociología, la antropología y, por supuesto, la gerontología. Igualmente recibe aportes de la economía, la terapia ocupacional, la planificación urbana y la arquitectura (Wahl y Weisman, 2003).

Desde este enfoque, los trabajos indican que la persona de edad avanzada tiene menores capacidades de adaptación para enfrentar las presiones del ambiente residencial y urbano (Lawton, 1986). También se sabe que el envejecimiento humano lleva implícita una pérdida progresiva de capacidades físicas y psicológicas, como discapacidad y dependencia (Iwarsson, 
2005), lo que condiciona su proceso de adaptación para enfrentar las crecientes presiones del ambiente, sobre todo en ciudades con problemas graves de habitabilidad (García-Ballesteros y Jiménez, 2016).

El proceso de envejecimiento se presenta de forma diferente en cada contexto físico y social, como entornos rurales y urbanos (viviendas y barrios) (Golant, 1986). Sin embargo, la mayoría de los estudios al respecto provienen principalmente de países desarrollados (Burton, Mitchell y Stride, 2011; Clarke y Gallagher, 2013). Asimismo, en las ciudades las presiones ambientales físicas y sociales se incrementan, limitando las capacidades de adaptación de las personas adultas mayores al condicionar sus actividades de la vida cotidiana y sus relaciones sociales (Feldman y Steptoe, 2004; Lang, Llewellyn, Langa, Wallace y Melzer, 2008; OMS, 2016). En los ambientes urbanos degradados, las personas envejecidas son vulnerables a los problemas de exclusión social, discapacidad y dependencia, ya que presentan menos activos y estrategias para poder enfrentarlos (Smith, 2009). Asimismo, muchos de los problemas de adaptación que presentan las personas con discapacidad se deben a los efectos del ambiente, más que a sus limitaciones personales (Hahn, 1987). En América Latina los gobiernos locales tienen poca capacidad de respuesta para enfrentar el desafío de adaptar los ambientes urbanos problemáticos a una población que envejece en situación de vulnerabilidad social (Bello, 2013). Existe además un conocimiento limitado de las interacciones persona-ciudad y las estrategias de adaptación ambiental encaminadas a impulsar el envejecimiento activo y saludable en el lugar (Batistoni, 2014; Sánchez-González, 2015). Por lo mismo, es necesario profundizar sobre el proceso de adaptación ambiental que experimentan las personas adultas mayores con objeto de favorecer políticas de desarrollo social y de salud pública, así como diseños urbanos inclusivos orientados a propiciar ciudades amigables con las personas de edad avanzada (OMS, 2007).

El presente ensayo reflexiona, desde el enfoque de la gerontología ambiental, sobre las estrategias de adaptación de las personas adultas mayores a los entornos urbanos, con alusiones al contexto de las ciudades de América Latina y el Caribe. La metodología se basó en una revisión exhaustiva de la producción científica a partir de la consulta de artículos de revistas indexadas en Web of Science y en Scopus. Los resultados indican la existencia de problemas derivados del reduccionismo analítico y de la simplificación de los modelos teóricos sobre adaptación ambiental y envejecimiento. También, la desadaptación está determinada por las capacidades personales y sociales del individuo (exclusión social, discapacidad y dependencia) y, especialmente, por los problemas físico-sociales del entorno urbano. Asimismo, se proponen estrategias de adaptación ambiental para el envejecimiento activo en 
el lugar, a partir de la evaluación de los activos personales, así como de los atributos y funciones del ambiente urbano.

\section{Adaptación ambiental y envejecimiento}

Desde la década de 1930, algunos expertos, como Lewin (1951), intentaron explicar las conductas de los individuos a partir de la relación entre las necesidades personales y las características del ambiente para promover la satisfacción de dichas necesidades (Fernández-Ballesteros e Izal, 1990). Estudios posteriores indican que la comprensión de la interacción personaambiente exige una perspectiva integral y sociohistórica (Holahan, 1982). La adaptación ambiental en el envejecimiento debe por lo tanto implicar una aproximación a la evolución de los factores multidimensionales del ambiente físico-social que influyen en las personas y, a su vez, de las capacidades de éstas para afectar a su entorno a lo largo del tiempo.

En los años 1960 y 1970 el avance del envejecimiento de la población obligó a los gobiernos, sobre todo de países anglosajones como Estados Unidos, a adaptar sus recursos a las crecientes demandas de salud, sociales, asistenciales y residenciales de este grupo de edad. Asimismo, se impulsaron investigaciones encaminadas a comprender la relación entre la persona y el ambiente donde envejece. Precisamente, durante esos años se desarrolló la gerontología ambiental, una especialidad encaminada a conocer, analizar, modificar y optimizar la relación entre la persona que envejece y su entorno físico-social, que tiene aportaciones de disciplinas como la psicología, la medicina, la arquitectura, la geografía, la sociología y la antropología (Wahl y Weisman, 2003). También, en las últimas décadas las contribuciones científicas de este campo multidisciplinar han posibilitado un acercamiento a los entornos privados e institucionalizados, como viviendas particulares y residencias.

Entre las distintas teorías que tratan de explicar la relación entre el envejecimiento y el ambiente urbano, destacan aproximaciones desde el modelo ecológico del envejecimiento (Lawton y Nahemow, 1973; Lawton, 1986), que relaciona las capacidades funcionales de las personas mayores con las presiones del ambiente. $\mathrm{Al}$ respecto, este modelo determinista, que ha sido objeto de amplia discusión, plantea que las personas a medida que envejecen presentan mayores limitaciones funcionales, como problemas de discapacidad y dependencia, lo que compromete su capacidad de adaptación para enfrentar las crecientes presiones ambientales, como barreras arquitectónicas, tráfico e inseguridad ciudadana. 
Algunas teorías se han centrado en el concepto de adaptación o proceso de negociación en el envejecimiento, por el cual la persona ajusta su comportamiento a las características del entorno en relación con su capacidad funcional. Dicha adaptación es un mecanismo que posibilita acostumbrarse, de forma gradual o súbita, a los cambios en el ambiente a lo largo del tiempo. También, el grado de adaptación está determinado por los factores físicos y sociales del entorno y los medios con que cuenta la persona para enfrentarlos (Lawton, 1986). Asimismo, las personas de avanzada edad con problemas funcionales son más vulnerables a las presiones del entorno urbano (barreras arquitectónicas, tráfico), lo que favorece el riesgo de caídas y atropellos, limitando el envejecimiento activo en el lugar (Buffel, Phillipson y Scharf, 2012). Igualmente, el proceso de adaptación de las personas mayores está determinado por el contexto ambiental y las experiencias espaciales (positivas y negativas) de envejecer en el lugar, circunstancias que determinan su identidad y apego al lugar (Sánchez-González, 2009). Al respecto, se ha acumulado conocimiento relativo a la identidad ambiental en el envejecimiento, el significado del lugar y las experiencias espaciales asociadas a los cambios en el entorno propio o por la reubicación (Rowles y Bernard, 2013).

Las personas mayores acomodan, ajustan y reubican las presiones del ambiente físico-social de acuerdo con sus capacidades (Kahana, 1982; Lawton, 1986). De hecho, se evidencia la existencia de interacciones recíprocas entre la persona y el ambiente urbano, donde los adultos mayores pueden actuar como agentes de cambio y favorecer su adaptación, combatiendo la tradicional imagen estereotipada de sujetos dependientes (Sánchez-González, 2015). Precisamente, esta nueva perspectiva se encuentra en la base del diseño del ambiente en el envejecimiento y está enfocada a posibilitar la participación activa de las personas mayores en los procesos de construcción, conservación, rehabilitación y adaptación de edificios y espacios públicos.

Los estudios desde la gerontología ambiental han examinado los procesos de adaptación en diferentes escalas espacio-temporales, ya sea la ciudad o la región (macro) (Kalache y Plouffe, 2010), el barrio (meso), y la vivienda y la residencia (micro). Aquí, el ambiente ha sido clasificado en: entorno físicoobjetivo (evaluación por sistema métrico decimal) y físico-subjetivo (evaluación por significado), entorno personal (familia y amigos), entorno de pequeños grupos (residencias, centro de trabajo), entorno suprapersonal (edificio de viviendas, barrios) y entorno social (leyes, normas) (Lawton, 1989). Igualmente, los resultados de los procesos de adaptación a diferente escala espaciotemporal son significativamente distintos. Por ello, desde una perspectiva social las escalas de análisis de la adaptación ambiental en el envejecimiento pueden ser personal, familiar y comunitaria (Wahl y Weisman, 2003). 
El proceso de adaptación a los centros residenciales de personas adultas mayores dependientes y los efectos de la institucionalización han sido analizados (Leturia, 1999), evidenciando sus complejas negociaciones con el ambiente físico-social y las dificultades de sentirse como en el hogar (Wilson, 2007). Otros trabajos han estudiado el proceso de adaptación de las personas que envejecen en el hogar, especialmente con problemas de discapacidad (Lansley et al., 2004; Newcomer, Kang, LaPlante y Kaye, 2005; Hsu y Tung, 2011; Thordardottir et al., 2015), observando diferencias entre las tipologías de casa y departamento, además de una relación desigual en el significado y adaptación a los espacios interiores de la vivienda (Rubinstein, 1989; Rowles, 1991).

El abordaje de la adaptación incluye la evaluación de las características del ambiente urbano, como la localización del barrio, la accesibilidad a los espacios públicos, la calidad de los servicios básicos, el transporte y el tráfico, y la exposición a peligros antrópicos (contaminación e inseguridad) y a peligros naturales (inundaciones y terremotos). En el envejecimiento el proceso de adaptación puede verse comprometido por la inadecuación de los entornos urbanos a los crecientes peligros naturales, como olas de calor, derivados de los efectos adversos del calentamiento global. A pesar de ello, a la fecha existe un limitado conocimiento desde la gerontología ambiental sobre los mecanismos de adaptación de las personas mayores ante el reto del cambio climático. Los resultados de las nuevas investigaciones están permitiendo conocer los efectos de las olas de calor sobre la morbilidad y la mortalidad de las personas adultas mayores (Ogg, 2005). Asimismo, se ha evidenciado que los problemas del entorno físico-social de la vivienda y el barrio condicionan el proceso de adaptación de las personas mayores con problemas de discapacidad, expuestas a riesgos de inundación en ciudades de América Latina y el Caribe (como Monterrey, México) (Chávez y SánchezGonzález, 2016).

La adaptación ambiental puede verse comprometida por factores físicos y sociales del entorno, así como por las capacidades de las personas mayores. En general, se pueden diferenciar dos procesos de adaptación: adaptativos y desadaptativos (Esquema 1). Al respecto, el proceso adaptativo describe un óptimo ajuste entre el entorno y la persona que envejece. En consecuencia, la adaptación es óptima, principalmente en entornos controlados o institucionalizados, donde el proceso de arreglo tiene una estructura y secuencia específicas (Leturia, 1999). A la inversa, el proceso desadaptativo implica desajustes entre el ambiente y la persona mayor. 


\section{Esquema 1}

Proceso de adaptación ambiental

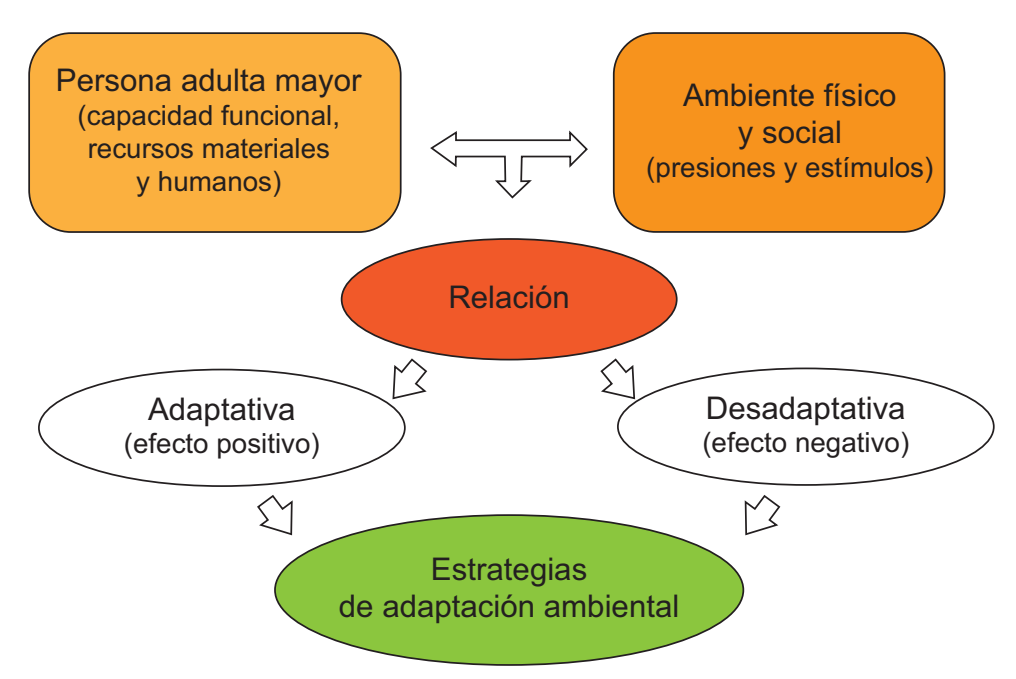

Fuente: Elaboración propia a partir de Lawton y Nahemow, 1973; Kahana, 1982; Lawton, 1986; Rowles, 1991; Golant, 2015.

\section{Procesos desadaptativos en el envejecimiento}

Diferentes investigaciones han abordado los factores explicativos de los procesos desadaptativos del envejecimiento en ambientes urbanos. En Latinoamérica la desigualdad social, asociada al crecimiento urbano no planeado, agrava las condiciones de habitabilidad y limita las capacidades de adaptación de las personas mayores, afectando negativamente su calidad de vida (Gómez et al., 2010; Sánchez-González, 2015). Ejemplos de ello, Ciudad de México y Rio Grande do Sul (Brasil) han registrado importantes problemas de urbanización asociados al rápido crecimiento demográfico, el cual derivó del proceso migratorio. Precisamente, una parte importante de la población envejecida urbana tiene un origen rural, lo que condiciona su distribución socioespacial y su menor red de apoyo familiar (Jasso et al., 2011). Esta circunstancia explica el envejecimiento demográfico de los barrios marginales de los centros históricos y de las periferias urbanas, como La Habana (Cuba) y Aguascalientes (México), lo que se traduce en que las personas mayores están más expuestas al riesgo de pobreza y exclusión 
social, así como a dificultades de acceso a servicios y equipamientos básicos (Narváez, 2011; Bello, 2013).

El estatus social del barrio suele condicionar el proceso de adaptación $\mathrm{y}$, en general, la calidad de vida de las personas mayores. Al respecto, un bajo estatus del barrio se asocia con menores inversiones en el espacio público, lo que favorece su desatención, el deterioro del mobiliario urbano, la presencia de barreras arquitectónicas y la ausencia de áreas verdes (Feldman y Steptoe, 2004). Por ejemplo, en los barrios marginales de las ciudades de Bogotá (Colombia) y de Florianópolis (Brasil), la percepción de alta inseguridad ciudadana y la menor presencia de áreas verdes desalientan entre las personas jubiladas la realización de actividades al aire libre, como caminar (Parra et al., 2010; Corseuil et al., 2011).

En el espacio público las barreras arquitectónicas aumentan los problemas de accesibilidad y aislamiento social de las personas de edad avanzada, sobre todo con problemas de discapacidad, agravando su discriminación (Avineri et al., 2012; Eronen et al., 2014). En los barrios degradados la existencia de barreras arquitectónicas favorece el riesgo de caídas y atropellos (Ståhl y Berntman, 2007; Avineri et al., 2012), e incrementa el aislamiento social y el miedo a salir de la casa (Delbaere et al., 2004; Rantakokko et al., 2017). Como consecuencia, el confinamiento de las personas mayores en el domicilio suele conllevar problemas de soledad, depresión y ansiedad (Routasalo et al., 2006; Hovbrandt et al., 2007; Cohen et al., 2009; Clarke y Gallagher, 2013). En la ciudad de Bahía Blanca (Argentina) se comprobó que las personas ancianas socialmente aisladas presentan mayor riesgo de deterioro funcional y malnutrición (Bagnulo y Pizarro, 2010).

Algunos autores consideran que las personas mayores tienen procesos de adaptación desiguales en ambientes similares (Filiberto et al., 2009), pero también el espacio público les condiciona porque no ofrece las mismas oportunidades sociales a las mujeres, los migrantes y los discapacitados. La adaptación de la persona mayor difiere en función de sus capacidades funcionales, como grado de discapacidad y dependencia, así como de su actitud y disponibilidad de activos y estrategias para ajustarse a las presiones del entorno urbano (Phillips et al., 2013). Asimismo, el propio sistema de oportunidades los segrega o puede intensificar las formas de opresión y discriminación (Soja, 2010; Phillipson, 2015). Por ejemplo, en la Ciudad de México los procesos de segregación socioespacial urbana, inherentes con la fragmentación social de la ciudad y con la formación histórica de enclaves urbanos, se asocian con la vulnerabilidad de la población envejecida para enfrentar las presiones ambientales (naturales y antrópicas), debido a sus recursos físicos, sociales y materiales limitados y al impacto de las políticas 
sociales, que amplifican o minimizan las diferencias socioespaciales en el envejecimiento urbano (Zamorano et al., 2012). Otro ejemplo: en la mayoría de las urbes latinoamericanas, como Santiago de Chile, el transporte público no se ajusta a las necesidades de la población mayor y constituye un elemento de exclusión social (Gajardo et al., 2012).

En el Esquema 2 se muestra el proceso de adaptación ambiental negativa o relación desadaptativa, que surge de la correspondencia excluyente entre la persona adulta mayor con problemas de discapacidad y las altas presiones ambientales (barreras arquitectónicas, tráfico, ausencia de áreas verdes, inseguridad ciudadana), donde el entorno urbano se percibe como un medio hostil que desalienta las actividades cotidianas fuera del hogar y que propicia el confinamiento.

\section{Estrategias de adaptación a los entornos urbanos en el envejecimiento}

En las últimas décadas se han desarrollado distintos modelos encaminados a formular estrategias de adaptación ambiental en el envejecimiento. En el Esquema 3 se señalan estrategias de adaptación ambiental para las personas adultas mayores, como la proactividad, la habilidad ambiental, el cambio de hábitos y costumbres, el empoderamiento, el estímulo ambiental o la reubicación. El modelo de proactividad ambiental enfatiza que las distintas capacidades de las personas mayores pueden representar posibilidades de adaptación a los diversos entornos (Lawton, 1986 y 1989), a través de modificarlos o participar en su transformación. También se argumenta que la persona mayor puede establecer estrategias para adaptarse al ambiente y manejar las posibles amenazas, convirtiéndose en un agente de cambio con una relación bidireccional con el entorno. Otro modelo es el de adaptación proactiva correctiva y preventiva, mismo que plantea que la persona mayor puede desarrollar comportamientos que se ajusten a los cambios en el entorno (Kahana y Kahana, 1996).

La bibliografía especializada constata que los ambientes determinan amplios aspectos de la vida cotidiana, como el bienestar físico y psicológico, así como seguridad, confort, apoyo social e identidad. De este modo, se establecen lazos afectivos con el lugar que habitamos, sobre todo durante el envejecimiento. Al respecto, Rowles (1991) indica que la relación persona-ambiente produce efectos positivos y negativos, los cuales pueden ser compensados o minimizados por medio de las experiencias espaciales, el apego y la fantasía. El conocimiento que brindan las experiencias espaciales 


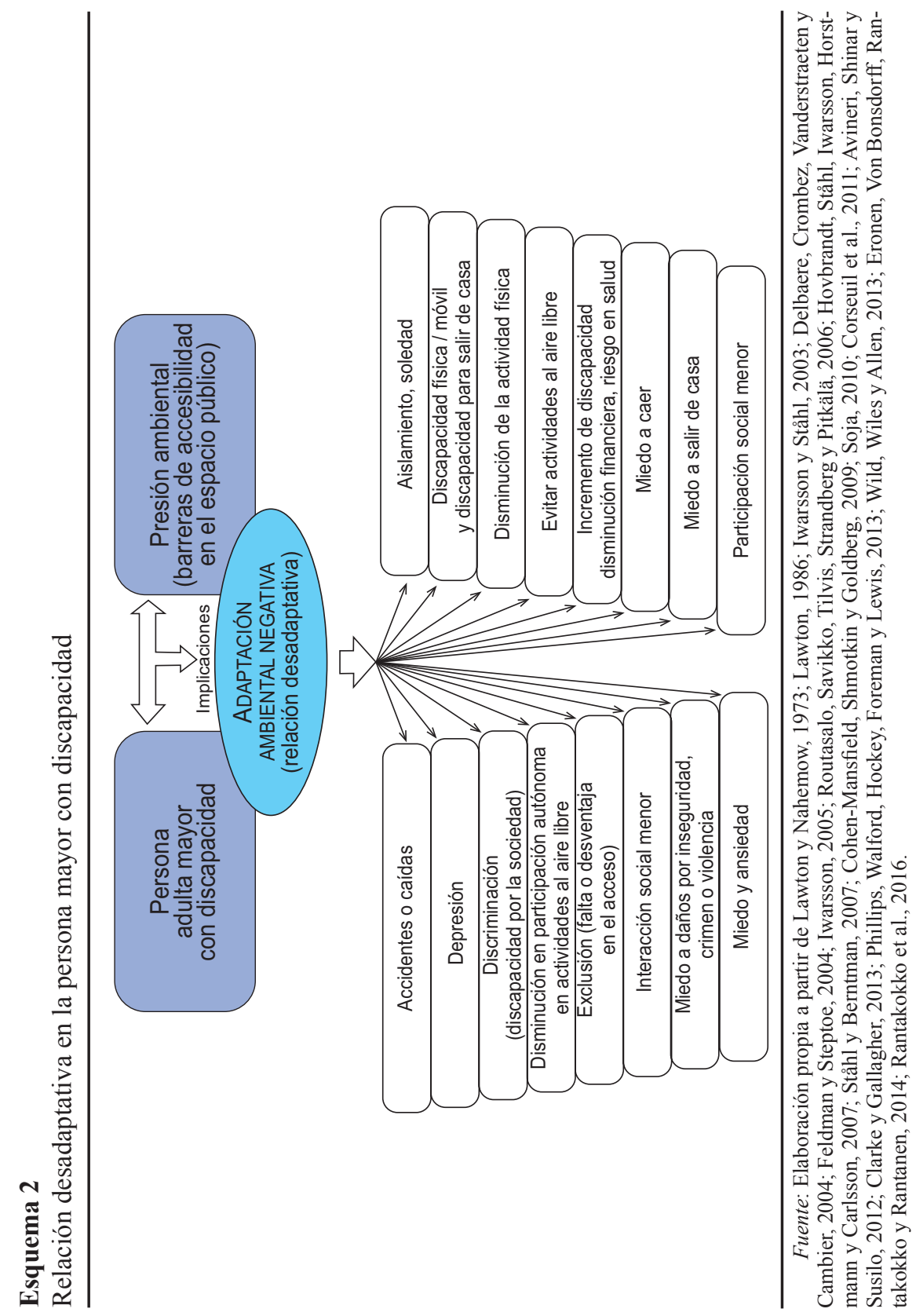




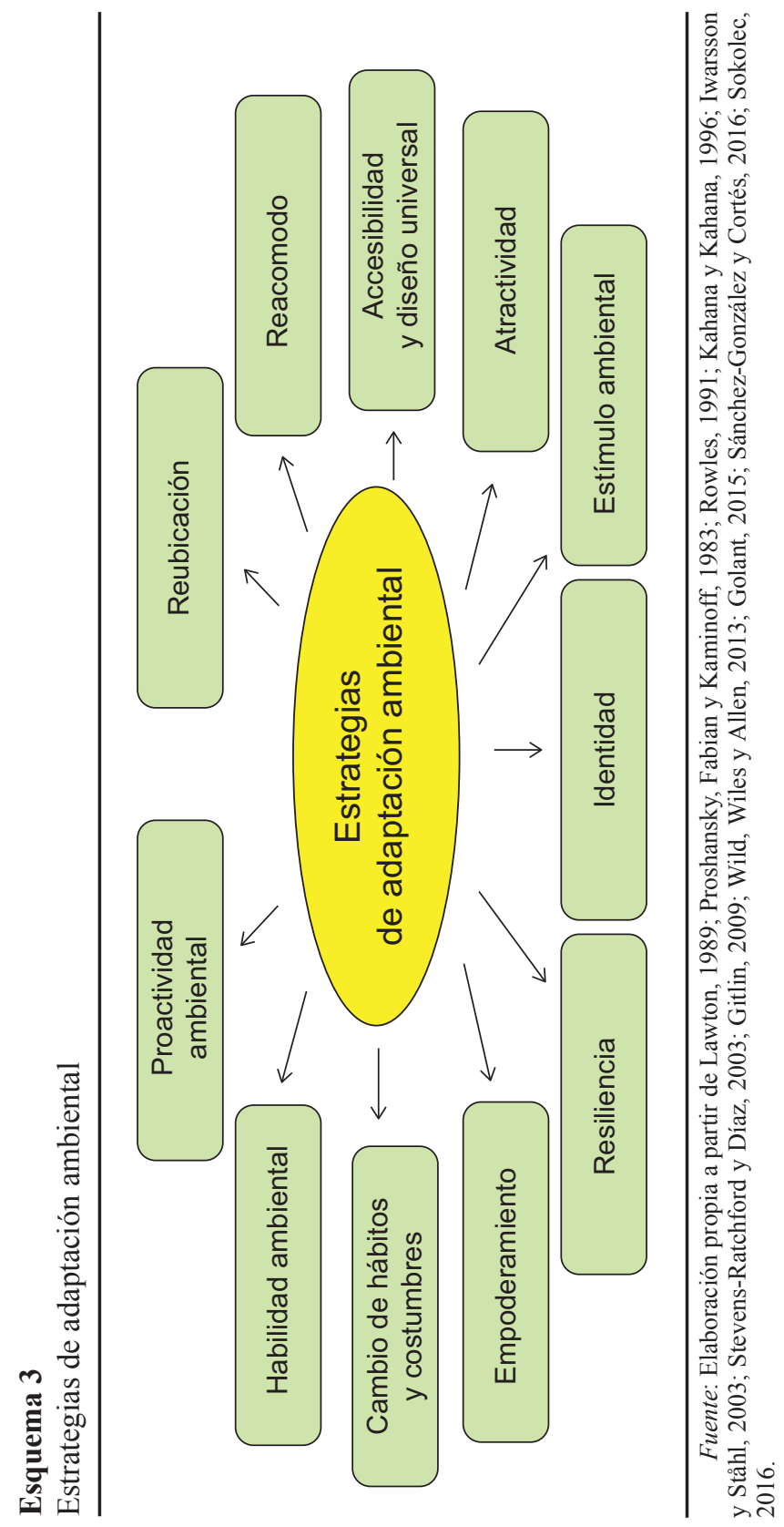


personales y de la comunidad (familiares y amigos) puede implicar cambios en los hábitos y costumbres para favorecer la adaptación.

Asimismo, las experiencias espaciales vinculadas con el lugar, y asociadas con aspectos sociales, culturales, religiosos y políticos, favorecen los procesos de adaptación, reduciendo el riesgo de la reubicación (SánchezGonzález, 2009). También las experiencias cotidianas (positivas y negativas) permiten comprender e interpretar el significado y satisfacción del hogar (Oswald, Hieber, Wahl y Mollenkopf, 2005). Por ejemplo, en la Ciudad de México se observa que las representaciones sociales positivas de la urbe se asocian con el apego y favorecen el envejecimiento activo (Alba, 2017). En Santiago de Chile el significado del uso del transporte público se relacionó con el mantenimiento de la autonomía y la identidad (Gajardo et al., 2012).

El empoderamiento de la persona mayor se vislumbra como una importante estrategia de adaptación, donde se enfatiza la confianza en su fortaleza y capacidad de gestión de los recursos para adecuarse al entorno físico y social (Stevens-Ratchford y Díaz, 2003; Sokolec, 2016). Igualmente, la resiliencia se ha considerado como un mecanismo que permite a una persona superar circunstancias traumáticas, como caídas, atropellos, abuso y violencia, y que posibilita su proceso de adaptación para envejecer en el lugar (Wild, Wiles y Allen, 2013). En la ciudad de Valparaíso (Chile), el deterioro de las calles y la falta de servicios, derivados de la falta de competencia de las autoridades, son compensadas por la actitud proactiva de las personas mayores para identificarse y adaptarse a su entorno con apoyo de las redes sociales (Fadda y Cortés, 2009).

El comportamiento adaptativo de las personas con limitaciones funcionales y el uso de dispositivos de apoyo para caminar, como bastón, andador o silla de ruedas, requiere de estrategias específicas de adaptación ambiental, a partir de cambios en el entorno físico-construido (adecuación de la vivienda y el barrio, incorporación de equipamientos y tecnología) y en el entorno social (entrenamiento de cuidadores) (Iwarsson y Ståhl, 2003). En materia de espacios públicos existen necesidades prioritarias como incorporar equipamientos y servicios adaptados (asientos y baños públicos), incrementar la seguridad e igualdad (eliminación de barreras arquitectónicas) (Hovbrandt, Ståhl, Iwarsson, Horstmann y Carlsson, 2007) e incorporar nueva tecnología en la señalización e iluminación (semáforos inteligentes) adaptada para personas con discapacidad (Phillips, Walford, Hockey, Foreman y Lewis, 2013).

Los adultos mayores tienden a reducir su espacio cotidiano al ámbito de la vivienda y el barrio, limitando sus recorridos y aumentando el tiempo en el hogar. Esta circunstancia convierte a los barrios en los lugares centrales 
del envejecimiento y en sus principales contextos de socialización (Membrado, 2010). De ahí la importancia de incorporar la planificación urbana gerontológica y el diseño universal en la generación de espacios públicos que favorezcan el envejecimiento activo en el lugar (Kalache y Plouffe, 2010; Iecovich, 2014). El diseño universal coadyuva a promover una relación adaptativa de la persona envejecida con su contexto urbano cotidiano (Iwarsson y Ståhl, 2003), contribuyendo a que permanezca de forma independiente, segura y activa (Michael, Green y Farquhar, 2006). El programa de ciudades amigables con las personas de edad (OMS, 2007) ha propiciado el desarrollo de estrategias de adaptación de espacios urbanos para el envejecimiento activo, mediante la introducción de nuevos diseños (edificios y espacios públicos), la incorporación de nuevos servicios y equipamientos (transporte público accesible) y la promoción de servicios de proximidad y ayuda a domicilio. Precisamente, la accesibilidad es un factor determinante de la calidad de vida de las personas de edad, ya que facilita su movilidad e independencia (Burton, Mitchell y Stride, 2011) y posibilita el equilibrio entre sus capacidades funcionales y las demandas del entorno (Kalache y Plouffe, 2010; Buffel et al., 2012).

El diseño de estrategias de adaptación ambiental debe involucrar la evaluación de las necesidades y capacidades de la persona mayor, así como las propiedades y características del entorno (Gitlin, 2009). Distintas investigaciones han señalado la importancia de analizar los factores ambientales y personales que posibilitan el óptimo ajuste entre el entorno y el envejecimiento. Al respecto, se ha planteado la evaluación de los elementos del ambiente físico-social y de las habilidades personales (control, competencia y comprensión), encaminadas a modificar y ajustarse al entorno (Proshansky, Fabian y Kaminoff, 1983). Además, el análisis de las capacidades funcionales personales en relación con los atributos (confort, orientación, privacidad) y funciones (seguridad, interacción social, familiaridad) del entorno pueden promover procesos de adaptación en el envejecimiento (Wahl y Gitlin, 2007).

Una de las estrategias de adaptación más prometedoras se centra en el diseño de ambientes estimulantes para favorecer el envejecimiento activo en el lugar. Al respecto, los estímulos ambientales pueden tener un origen natural (paisajes, áreas verdes, flora y fauna) o antrópico (música, colores y formas), además pueden mejorar las capacidades personales y reducir las anormalidades ambientales (Kweon, Sullivan y Wiley, 1998; Wiles, Leibing, Guberman, Reeve y Allen, 2011). De hecho, la exposición a un ambiente enriquecido produce una mayor estimulación que podría restaurar la función neuronal en personas con demencias (alzhéimer, párkinson) (Hannan, 2014). También se ha subrayado la importancia de la estimulación ambiental en el 
proceso de adaptación de las personas mayores al entorno cotidiano, destacando la necesidad de diseñar viviendas y barrios más accesibles, equipados y estimulantes (Lawton, 1986). En Aguascalientes, México, la atractividad del mercado público tradicional, determinada por la funcionalidad de los atributos del ambiente físico-construido y la usabilidad de las funciones del entorno social, promueve estrategias de adaptación e impulsa el envejecimiento activo y saludable (Sánchez-González y Cortés, 2016).

\section{Discusión}

Las contribuciones científicas desde la gerontología ambiental han posibilitado un acercamiento limitado a las relaciones entre la persona que envejece y el ambiente urbano, sobre todo en las regiones en desarrollo, como América Latina y el Caribe, donde el envejecimiento demográfico urbano plantea importantes retos sociales, de salud y residenciales a los gobiernos locales. En las últimas décadas se ha producido un interés creciente por analizar los factores explicativos de los procesos desadaptativos en el envejecimiento, donde se argumenta la existencia de un conflicto entre quienes deciden la planificación de las ciudades y quienes la viven y representan (Vega, 2014). Asimismo, existe un conocimiento limitado de los factores que posibilitan estrategias adaptativas encaminadas a alcanzar un óptimo ajuste entre el entorno urbano y la persona mayor. $\mathrm{Al}$ respecto, la progresiva tendencia al reduccionismo analítico ha simplificado y empobrecido los modelos teóricos explicativos de la relación ambiente urbano-persona, lo que ha dificultado la comprensión de los desafíos ambientales que enfrenta la ciudadanía que envejece, como la urbanización acelerada, la globalización y el cambio climático. Algunos modelos teóricos explican el concepto de adaptación ambiental en un momento determinado, sin considerar el efecto generacional o de tiempo pasado y presente. Este aspecto importante compromete el conocimiento del comportamiento adaptativo presente de la persona adulta mayor en el entorno urbano, que puede estar en función de sus actividades o conductas en el pasado (Fernández-Ballesteros e Izal, 1990).

En el envejecimiento el proceso de adaptación está determinado, sobre todo, por las limitaciones funcionales (físicas y psicológicas) de los sujetos para enfrentar las presiones ambientales de origen natural y social en el entorno urbano. Los factores limitantes personales, como enfermedades degenerativas, discapacidad y dependencia, pueden hacer la diferencia en esta etapa de la vida (Satariano, 2006). Las posibilidades de adaptación al entorno físico y social en el envejecimiento están condicionadas por el con- 
texto natural, social, económico, político, cultural y religioso. Igualmente, los procesos de adaptación ambiental pueden ser distintos según las características del ambiente urbano y las capacidades personales en términos de salud, economía y cultura. Las personas que cuenten con activos (económico, apoyo social) estarán en mejor disposición de adaptarse o de adecuar su entorno a sus necesidades (Smith, 2009).

El avance limitado del conocimiento sobre las estrategias de adaptación al entorno urbano en el envejecimiento se relaciona con la existencia de estudios centrados en ambientes generalmente de países anglosajones (Rubinstein, 1989; Rowles, 1991). Tales investigaciones suelen carecer de análisis exhaustivos de los factores físicos y sociales determinantes del proceso de adaptación ambiental, como las redes de apoyo, las barreras arquitectónicas o la atractividad del ambiente. Los resultados y propuestas de dichas investigaciones no se adecuan a los contextos citadinos de las ciudades latinoamericanas. Por ello, es necesaria una reflexión pausada y una revisión exhaustiva de los programas de otros contextos internacionales, cuyos supuestos beneficios suelen justificar costosos abordajes para su implementación, aunque los resultados pudieran no ser los deseados y comprometer con ello el éxito del envejecimiento activo en estos espacios. Las diferentes escalas de análisis espacio-temporales de las estrategias de adaptación utilizadas en los estudios aquí reportados, pueden generar resultados significativamente distintos.

En la región latinoamericana la urbanización no planeada y las desigualdades sociales generan procesos desadaptativos que limitan el envejecimiento en el lugar (Sánchez-González, 2015), como condicionar sus actividades de la vida cotidiana, sus relaciones y participación sociales (Fadda y Cortés, 2009; Corseuil et al., 2011; Phillips et al., 2013). De hecho, las políticas sociales limitadas no contrarrestan los efectos de la segregación espacial urbana y los problemas graves de desigualdad social que aquejan a la población envejecida, como la pobreza y la exclusión social (Smith, 2009). Los programas sociales no han tenido el éxito deseado debido a la existencia de zonas urbanas amplias con índices de marginación altos, habitadas por elevados porcentajes de personas mayores vulnerables, con problemas de exclusión social, discapacidad y dependencia, así como importantes dificultades de acceso a las limitadas ayudas y apoyos gubernamentales (Bello, 2013). Al respecto, en América Latina y el Caribe los retos del envejecimiento demográfico urbano deben implicar una mayor comprensión de los factores determinantes de la vulnerabilidad y, sobre todo, un exhaustivo conocimiento de las negociaciones que se establecen entre la persona mayor y el entorno urbano, encaminado a reducir las presiones ambientales y la mejora de la 
capacidad funcional para propiciar estrategias de adaptación ambiental (Tomasini, 2005).

Como resultado de la revisión de la bibliografía especializada y de la reflexión sobre la necesidad de favorecer programas encaminados al desarrollo de estrategias de adaptación ambiental en el envejecimiento activo en ciudades de América Latina y el Caribe, mediante la evaluación de los activos personales, de los atributos y funciones del ambiente urbano, a continuación se exponen algunas propuestas de diseño de estrategias de adaptación ambiental en el envejecimiento y las posibles limitaciones para su desarrollo en la región:

1. Diseñar ciudades amigables para las personas de edad, que posibiliten el envejecimiento activo y faciliten su adaptación al entorno cotidiano (OMS, 2007). En Latinoamérica la mayoría de los intentos llevados a cabo por los gobiernos locales para sumarse al programa de ciudades amigables han resultado infructuosos, dada la falta de continuidad en el compromiso y, sobre todo, el escaso apoyo político y económico para la consecución de tales fines (Chávez y Sánchez-González, 2016). Por ello, se recomienda que la propuesta vaya acompañada de un nuevo marco normativo que asegure su protección y continuidad.

2. Promover ambientes estimulantes para el envejecimiento activo en el lugar, a través de nuevos diseños residenciales y paisajes urbanos más provocadores, acompañados del fomento de espacios públicos atractivos, como parques y áreas verdes, además de edificios con elementos naturales. Precisamente, estos nuevos diseños incorporan estímulos visuales y sensoriales que contribuyen a reducir los niveles de estrés y el deterioro cognitivo asociado al proceso de envejecimiento (Mo et al., 2015). Asimismo, estos contextos estimulantes tienen una función terapéutica al promover estilos de vida saludables entre las personas mayores, como actividades al aire libre (deporte, caminar), contribuyendo a reducir la prevalencia de enfermedades y a mejorar su calidad de vida (Hannan, 2014). Al respecto, es necesario fomentar la investigación sobre espacios públicos estimulantes y atractivos para el envejecimiento en la región, a partir de la evaluación de las características físico-sociales de los entornos y la percepción de los sujetos, la cual está condicionada por experiencias personales y factores socioculturales.

3. Incentivar la incorporación de la gerontotecnología (aplicación de tecnología en el área de la gerontología) y la domótica (automatización 
de la vivienda) en los entornos residenciales y urbanos. Los avances tecnológicos brindan autonomía a las personas mayores dependientes (Hwang, Cummings, Sixsmith y Sixsmith, 2011), sin embargo están condicionados por los limitados activos (recursos económicos propios o de terceros) y las estrategias de las personas mayores (Lichtenberg, MacNeill y Mast, 2000; Richardson et al., 2015).

4. Facilitar la reubicación en determinadas circunstancias personales y ambientales, como personas frágiles y entornos degradados (Golant, 2015). El cambio de residencia para las personas mayores puede estar motivado por la pérdida de autonomía y la necesidad de recibir cuidados (Caprón y González, 2010; García-Puebla, Román, Alemán y Valenzuela, 2015). Por ello, la reubicación se convierte en una estrategia necesaria para las personas muy vulnerables, con alto grado de discapacidad y dependencia, en riesgo de abandono y residentes en ambientes problemáticos (viviendas precarias y barrios marginales). Empero, en la región los escasos recursos destinados a las políticas sociales de atención al adulto mayor limitan las opciones de la reubicación, incluso en casos de extrema gravedad, como en los casos de personas enfermas encamadas que viven solas y necesitan cuidados continuos.

\section{Reflexiones finales}

La revisión bibliográfica aquí expuesta nos muestra que los avances en la comprensión de las estrategias de adaptación ambiental para favorecer el envejecimiento activo en el lugar de residencia, se han desarrollado, sobre todo, en ciudades de países anglosajones. La mayoría de las teorías sobre adaptación ambiental se han abordado parcialmente y se ha otorgado prioridad a ciertos temas, como los efectos de la institucionalización y las adaptaciones a la vivienda. Asimismo, se constata una falta de comunicación en este ámbito y la tendencia hacia un reduccionismo analítico, surgidas en contextos internacionales y que condicionan las líneas de investigación que tienen prioridad en la región de América Latina y el Caribe.

Las investigaciones se han desarrollado, principalmente, en ambientes controlados, como hospitales, residencias y viviendas, donde han primado los enfoques de disciplinas de la salud y la asistencia social. Durante años, este hecho ha condicionado las metodologías y el conocimiento que se tiene de la adaptación ambiental en el envejecimiento. Así, otros entornos culturales vinculados con ambientes rurales y urbanos de países en desarrollo han 
sido poco abordados. También se tiene un escaso conocimiento sobre las estrategias de adaptación de las personas mayores en entornos urbanos ante los efectos adversos del cambio climático. Por tanto, los organismos internacionales deben priorizar los procesos de adaptación de las personas ancianas a los distintos ecosistemas, a partir de enfoques interdisciplinares con nuevas aportaciones de disciplinas como arquitectura, diseño, geografía y antropología.

El futuro de las investigaciones sobre la adaptación ambiental en el envejecimiento requiere de estudios internacionales con enfoques longitudinales, ya que son cruciales para el desarrollo de políticas de salud, sociales y residenciales enfocadas a mejorar la calidad de vida de las personas mayores. Estos trabajos pueden contribuir a entender los procesos de adaptación de los adultos mayores en distintos escenarios urbanos a lo largo del tiempo. También es importante continuar con la comprensión de la identidad y el apego al lugar en el envejecimiento, así como profundizar en el análisis exhaustivo de los atributos y funciones que definen la compleja relación persona-ambiente. Para ello, es imprescindible la incorporación de escalas espacio-temporales de análisis, así como desarrollar nuevos métodos y herramientas de investigación, que incluyan los aspectos psicosociales y culturales de las personas envejecidas.

En la región, la incertidumbre sobre el futuro de los millones de personas que envejecen en las ciudades está asociada con la capacidad para generar ambientes amigables, seguros y estimulantes para vivir. En las urbes de América Latina las personas mayores experimentan una situación de vulnerabilidad que demanda respuestas urgentes de los gobiernos locales y regionales. Asimismo, esta nueva realidad exige una mayor conciencia social sobre los retos del envejecimiento de la población urbana en las próximas décadas y el desarrollo de programas de envejecimiento activo. Precisamente, nuestra inquietud radica en la capacidad y el tiempo de respuesta para adaptarnos a los desafíos demográficos y ambientales en el siglo XXI.

\section{Bibliografía}

Alba, M. (2017). Representaciones sociales y experiencias de vida cotidiana de los ancianos en la Ciudad de México. Estudios Demográficos y Urbanos, 32(1), 9-36. Recuperado de http://estudiosdemograficosyurbanos. colmex.mx/index.php/edu/article/view/1616/pdf

Avineri, E., Shinar, D. y Susilo, Y. O. (2012). Pedestrians' behaviour in cross walks: The effects of fear of falling and age. Accident Analysis \& Pre- 
vention, 44(1), 30-34. Recuperado de https://www.sciencedirect.com/ science/article/pii/S0001457510003726

Bagnulo, C. y Pizarro, N. (2010). Distribución espacial de los adultos mayores en condición de riesgo de malnutrición en la ciudad de Bahía Blanca. Revista Universitaria de Geografia, 19(1), 119-138. Recuperado de http://www.scielo.org.ar/scielo.php?script=sci_arttext\&pid=S1 852-42652010000100009

Batistoni, S. (2014). Gerontologia ambiental: panorama de suas contribuições para a atuação do gerontólogo. Revista Brasileira de Geriatria e Gerontologia, 17(3), 647-657. Recuperado de http://www.scielo.br/pdf/rbgg/ v17n3/1809-9823-rbgg-17-03-00647.pdf

Bello, W. (2013). Vulnerabilidad sociodemográfica de las personas de la tercera edad. Estudio de caso: centro histórico La Habana. Cuadernos Geográficos, 52(1), 153-177. Recuperado de http://revistaseug.ugr.es/index. php/cuadgeo/article/view/930/1104

Buffel, T., Phillipson, C. y Scharf, T. (2012). Ageing in urban environments: Developing 'age-friendly' cities. Critical Social Policy, 32(4), 597-617. Recuperado de http://journals.sagepub.com/doi/pdf/10.1177/026101 8311430457

Burton, E. J., Mitchell, L. y Stride, C. B. (2011). Good places for ageing in place: Development of objective built environment measures for investigating links with older people's wellbeing. BMC Public Health, 11(1), 1-13. Recuperado de https://bmcpublichealth.biomedcentral.com/articles /10.1186/1471-2458-11-839

Capron, G. y González, S. (2010). Movilidad residencial de los adultos mayores y trayectorias de vida familiares en la Zona Metropolitana del Valle de México. Alteridades, 39, 67-78. Recuperado de http://www. scielo.org.mx/pdf/alte/v20n39/v20n39a6.pdf

Chávez, R. y Sánchez-González, D. (2016). Envejecimiento vulnerable en hogares inundables y su adaptación al cambio climático en ciudades de América Latina: el caso de Monterrey. Papeles de Población, 22(90), 9-42. Recuperado de http://www.redalyc.org/pdf/112/11249884002.pdf

Clarke, P. y Gallagher, N. A. (2013). Optimizing mobility in later life: The role of the urban built environment for older adults aging in place. Journal of Urban Health, 90(6), 997-1009. Recuperado de https://www. ncbi.nlm.nih.gov/pmc/articles/PMC3853178/

Cohen-Mansfield, J., Shmotkin, D. y Goldberg, S. (2009). Loneliness in old age: Longitudinal changes and their determinants in an Israeli sample. International Psychogeriatrics, 21(06), 1160-1170. Recuperado https:// www.ncbi.nlm.nih.gov/pubmed/19785916 
Consejo Nacional de Población (Conapo). (2010). Proyecciones de la población de México, 1990-2010, 2010-2050. México. Recuperado de http:// www.conapo.gob.mx/es/CONAPO/Proyecciones (25 de agosto de 2017).

Corseuil, M. W., Schneider, I. J. C., Silva, D. A. S., Costa, F. F., Silva, K. S., Borges, L. J. y d'Orsi, E. (2011). Perception of environmental obstacles to commuting physical activity in Brazilian elderly. Preventive Medicine, 53(4), 289-292. Recuperado de https://www.sciencedirect.com/ science/article/pii/S0091743511002829

Delbaere, K., Crombez, G., Vanderstraeten, G., Willems, T. y Cambier, D. (2004). Fear-related avoidance of activities, falls and physical frailty. A prospective community-based cohort study. Age and Ageing, 33(4), 368-373. Recuperado de https://academic.oup.com/ageing/article/ $33 / 4 / 368 / 17936$

Eronen, J., von Bonsdorff, M., Rantakokko, M. y Rantanen, T. (2014). Environmental facilitators for outdoor walking and development of walking difficulty in community-dwelling older adults. European Journal of Ageing, 11(1), 67-75. Recuperado de https://www.ncbi.nlm.nih.gov/pmc/ articles/PMC5549184/

Fadda, G. y Cortés, A. (2009). Hábitat y adulto mayor: el caso de Valparaíso. Revista INVI, 24(66), 89-113. Recuperado de https://scielo.conicyt.cl/ scielo.php?script $=$ sci_arttext\&pid $=$ S0718-83582009000200003

Feldman, P. J. y Steptoe, A. (2004). How neighborhoods and physical functioning are related: The roles of neighborhood socioeconomic status, perceived neighborhood strain, and individual health risk factors. Annals of Behavioral Medicine, 27(2), 91-99. Recuperado de https://link.springer. com/content/pdf/10.1207\%2Fs15324796abm2702_3.pdf

Fernández-Ballesteros, R. e Izal, M. (1990). Modelos ambientales sobre la vejez. Anales de Psicología, 6(2), 181-198. Recuperado de http://www. um.es/analesps/v06/v06_2/07-06_2.pdf

Filiberto, D., Wethington, E., Pillemer, K., Wells, N., Wysocki, M. y Parise, J. T. (2009). Older people and climate change: Vulnerability and health effects. Generations, 33(4), 19-25. Recuperado de http://www.asaging. $\mathrm{org} / \mathrm{blog} /$ older-people-and-climate-change-vulnerability-and-healtheffects

Gajardo, J., Navarrete, E., López, C., Rodríguez, J., Rojas, A., Troncoso, S. y Rojas, A. (2012). Percepciones de personas mayores sobre su desempeño en el uso de transporte público en Santiago de Chile. Revista Chilena de Terapia Ocupacional, 12(1), 88-102. Recuperado de https:// revistaterapiaocupacional.uchile.cl/index.php/RTO/article/view/ $22055 / 23371$ 
García-Ballesteros, A. y Jiménez, B. C. (2016). Envejecimiento y urbanización: implicaciones de dos procesos coincidentes. Investigaciones Geográficas, 89, 58-73. Recuperado de http:/www.igg.unam.mx/sigg/ utilidades/docs/pdfs/publicaciones/inves_geo/boletines/89/preprint d__47362.pdf

García-Puebla, M. D., Román, R., Alemán, H. y Valenzuela, B. (2015). Identidades en transición: el caso de las personas adultas mayores. En S. Garay, M. C. Arroyo y J. E. Bracamontes (coords.), Vejez, familia y bienestar. Dimensiones micro y macrosociales del envejecimiento y la vejez (pp. 265-293). México: Universidad Autónoma de Nuevo León.

Gitlin, L. N. (2009). Environmental adaptations for older adults and their families in the home and community. En International handbook of occupational therapy interventions. Nueva York: Springer.

Golant, S. M. (1986). A place to grow old: The meaning of environment in old age. Nueva York: Columbia University Press.

Golant, S. M. (2015). Aging in the right place. Maryland: Health Professions Press.

Gómez, L. F., Hernández, A. y Parra, D. (2010). Ambientes urbanos y actividad física en adultos mayores: relevancia del tema para América Latina. Revista de Salud Pública, 12(2), 327-335. Recuperado de http:// www.scielo.org.co/pdf/rsap/v12n2/v12n2a16.pdf

Hahn, H. (1987). Adapting the environment to people with disabilities: Constitutional issues in Canada. International Journal of Rebabilitation Research, 10(4), 363-372.

Hannan, A. J. (2014). Environmental enrichment and brain repair: Harnessing the therapeutic effects of cognitive stimulation and physical activity to enhance experience-dependent plasticity. Neuropathology and Applied Neurobiology, 41(1), 13-25. Recuperado de https://onlinelibrary.wiley. com/doi/epdf/10.1111/nan.12102

Holahan, C. J. (1982). Environmental Psychology. Nueva York: Random House.

Hovbrandt, P., Ståhl, A., Iwarsson, S., Horstmann, V. y Carlsson, G. (2007). Very old people's use of the pedestrian environment: Functional limitations, frequency of activity and environmental demands. European Journal of Ageing, 4(4), 201-211. Recuperado de https://www.ncbi.nlm. nih.gov/pmc/articles/PMC5546367/

Hsu, H.-C. y Tung, H.-J. (2011). Coping strategies and adaptation for the disabled elderly in Taiwan. Geriatrics \& Gerontology International, 11(4), 488-495. Recuperado de https://onlinelibrary.wiley.com/doi/ epdf/10.1111/j.1447-0594.2011.00701.x 
Hwang, E., Cummings, L., Sixsmith, A. y Sixsmith, J. (2011). Impacts of home modification on aging-in-place. Journal of Housing for the Elderly, 25(3), 246-257.

Iecovich, E. (2014). Aging in place: From theory to practice. Anthropological Notebooks, 20(1), 21-33. Recuperado de https://pdfs.semanticscholar. org/f9fc/8e6e859408543cb512a499d37a4267edb348.pdf

Instituto Nacional de Estadística y Geografía (INEGI) (2015). Estadísticas a propósito del Día Internacional de las Personas de Edad (1 de octubre). México. Recuperado de: http://www.inegi.org.mx/saladeprensa/apropo sito/ 2016/edad2016_0.pdf (25 de agosto de 2017).

Iwarsson, S. (2005). A long-term perspective on person-environment fit and ADL dependence among older Swedish adults. The Gerontologist, 45(3), 327-336. Recuperado de https://academic.oup.com/gerontologist/article $/ 45 / 3 / 327 / 553157$

Iwarsson, S. y Ståhl, A. (2003). Accessibility, usability and universal design: Positioning and definition of concepts describing person-environment relationships. Disability and Rehabilitation, 25(2), 57-66.

Jasso, P., Montoya, J. y Cadena, E. (2011). Los adultos mayores en las zonas metropolitanas de México: desigualdad socioeconómica y distribución espacial, 1990-2005. Papeles de Población, 17(70), 81-124. Recuperado de http://www.redalyc.org/articulo.oa?id=11221584005

Kahana, E. (1982). A congruence model of person-environment interaction. En M. Powell Lawton, P. G. Windley y T. O. Byerts (eds.), Aging and the environment. Theoretical approaches (pp. 97-120). Nueva York: Springer.

Kahana, E. y Kahana, B. (1996). Conceptual and empirical advances in understanding aging well through proactive adaptation. En V. L. Bengtson (ed.), Adulthood and aging. Research on continuities and discontinuities (pp. 18-40). Nueva York: Springer Publishing Company.

Kalache, A. y Plouffe, L. (2010). Towards global age-friendly cities: Determining urban features that promote active aging. Journal of Urban Health, 87(5), 733-739. Recuperado de https://www.ncbi.nlm.nih.gov/ pmc/articles/PMC2937125/

Kweon, B.-S., Sullivan, W. C. y Wiley, A. R. (1998). Green common spaces and the social integration of inner-city older adults. Environment and Behavior, 30(6), 832-858. Recuperado de https://www.researchgate.net/ publication/249624259_Green_Common_Spaces_and_the_Social_ Integration_of_Inner-City_Older_Adults

Lang, I. A., Llewellyn, D. J., Langa, K. M., Wallace, R. B. y Melzer, D. (2008). Neighborhood deprivation and incident mobility disability in 
older adults. Age and Ageing, 37(4), 403-410. Recuperado de https:// academic.oup.com/ageing/article/37/4/403/40693

Lansley, P., McCreadie, C., Tinker, A., Flanagan, S., Goodacre, K. y TurnerSmith, A. (2004). Adapting the homes of older people: A case study of costs and savings. Building Research \& Information, 32(6), 468-483.

Lawton, M. P. (1986). Environment and ageing (2a ed.). Albany, NY: Centre for the Study of Ageing.

Lawton, M. P. (1989). Environmental proactivity and affect in older. En S. Spacapan y S. Oskamp (eds.), The social psychology of aging (pp. 135163). Newbury Park: Sage.

Lawton, M. P. y Nahemow, L. (1973). Ecology and the aging process. En C. Eisdorfer y M. P. Lawton (eds.), The psychology of adult development (pp. 619-674). Washington: American Psychological Association.

Lewin, K. (1951). Field theory in social science. En R. L. Rubinstein, M. Moss y M. H. Kleban (eds.), Selected theoretical papers. Nueva York: Harper \& Brothers.

Leturia, F. J. (1999). El proceso de adaptación en centros residenciales para personas mayores. Revista Española de Geriatría y Gerontología, 34(2), 105-112. Recuperado de http://www.elsevier.es/es-revista-revista-espa nola-geriatria-gerontologia-124-articulo-el-proceso-adaptacion-centrosresidenciales- 13011672

Lichtenberg, P. A., MacNeill, S. E. y Mast, B. T. (2000). Environmental press and adaptation to disability in hospitalized live-alone older adults. The Gerontologist, 40(5), 549-556. Recuperado de https://academic.oup. com/gerontologist/article/40/5/549/586997

Membrado, M. (2010). Experiencias de envejecer y experiencias urbanas: un estudio en el suroeste francés. Alteridades, 39, 57-65. Recuperado de http://www.scielo.org.mx/pdf/alte/v20n39/v20n39a5.pdf

Michael, Y. L., Green, M. K. y Farquhar, S. A. (2006). Neighborhood design and active aging. Health \& Place, 12(4), 734-740. Recuperado de https:// www.sciencedirect.com/science/article/pii/S1353829205000419

Mo, C., Hannan, A. J. y Renoir, T. (2015). Environmental factors as modulators of neurodegeneration: Insights from gene-environment interactions in Huntington's disease. Neuroscience and Biobehavioral Reviews, 52(1), 178-192. Recuperado de https://ac.els-cdn.com/S0149763415000731/1s2.0-S0149763415000731-main.pdf?_tid=a49c12f6-f792-466a-be4c680dea293a29\&acdnat $=1532373082 \_9 a b f e 0 d d a d 3376 c 9180 f f 889 b c 9$ da169

Narváez, Ó. L. (2011). Urbanismo gerontológico: envejecimiento demográfico y equipamiento urbano en Aguascalientes. Investigación y Ciencia, 
51, 16-24. Recuperado de http://www.redalyc.org/pdf/674/67418397003. pdf

Newcomer, R., Kang, T., LaPlante, M. y Kaye, S. (2005). Living quarters and unmet need for personal care assistance among adults with disabilities. The Journals of Gerontology Series B: Psychological Sciences and Social Sciences, 60(4), S205-S213. Recuperado de https://academic. oup.com/psychsocgerontology/article/60/4/S205/545306

Ogg, J. (2005). Heat wave. Londres: The Young Foundation.

OMS. (2007). Ciudades globales amigables con los mayores: una guía. Ginebra: Organización Mundial de la Salud. Recuperado de http://www. who.int/ageing/AFCSpanishfinal.pdf (25 de agosto de 2017).

OMS. (2016). Preventing disease through healthy environments: A global assessment of the burden of disease from environmental risks. París. Recuperado de http://apps.who.int/iris/bitstream/10665/204585/ 1/9789241565196_eng.pdf (25 de agosto de 2017).

ONU. (2015). En el día mundial de las ciudades la ONU destaca el papel del diseño urbano. Recuperado de http://www.un.org/spanish/News/ story.asp?NewsID=33728\#.VxwUZ9ThDIU (25 de agosto de 2017).

Oswald, F., Hieber, A., Wahl, H.-W. y Mollenkopf, H. (2005). Ageing and person-environment fit in different urban neighborhoods. European Journal of Ageing, 2(2), 88-97. Recuperado de https://link.springer.com/ article/10.1007/s10433-005-0026-5

Parra, D. C., Gomez, L. F., Sarmiento, O. L., Buchner, D., Brownson, R., Schimd, T., Gomez, V. y Lobelo, F. (2010). Perceived and objective neighborhood environment attributes and health related quality of life among the elderly in Bogota, Colombia. Social Science \& Medicine, 70(7), 1070-1076. Recuperado de https://www.sciencedirect.com/science/ article/pii/S0277953610000456

Phillips, J., Walford, N., Hockey, A., Foreman, N. y Lewis, M. (2013). Older people and outdoor environments: Pedestrian anxieties and barriers in the use of familiar and unfamiliar spaces. Geoforum, 47, 113-124. Recuperado de https://www.sciencedirect.com/science/article/pii/ S0016718513000742

Phillipson, C. (2015). Developing age-friendly urban communities: Critical issues for public policy. Public Policy \& Aging Report, 25(1), 4-8. Recuperado de https://academic.oup.com/ppar/article/25/1/4/1510304

Proshansky, H. M., Fabian, A. K. y Kaminoff, R. (1983). Place-identity: Physical world socialization of the self. Journal of Environmental Psychology, 3(1), 57-83.

Rantakokko, M., Portegijs, E., Viljanen, A., Iwarsson, S., Kauppinen, M. y 
Rantanen, T. (2017). Perceived environmental barriers to outdoor mobility and changes in sense of autonomy in participation outdoors among older people: A prospective two-year cohort study. Aging \& Mental Health, 21(8), 805-809.

Richardson, J., DePaul, V., Officer, A., Wilkins, S., Letts, L., Bosch, J. y Wishart, L. (2015). Development and evaluation of Self-management and Task-oriented Approach to Rehabilitation Training (START) in the home: Case report. Physical Therapy, 95(6), 934-943. Recuperado de https://academic.oup.com/ptj/article/95/6/934/2686388

Routasalo, P. E., Savikko, N., Tilvis, R., Strandberg, T. y Pitkälä, K. H. (2006). Social contacts and their relationship to loneliness among aged people. A population-based study. Gerontology, 52(3), 181-187. Recuperado de https://www.karger.com/Article/Abstract/91828

Rowles, G. D. (1991). Beyond performance: Being in place as a component of occupational therapy. American Journal of Occupational Therapy, 45(3), 265-271. Recuperado de https://ajot.aota.org/article.aspx?articleid=1876648

Rowles, G. D. y Bernard M. (eds.) (2013). Environmental gerontology: Making meaningful places in old age. Nueva York: Springer.

Rubinstein, R. L. (1989). The home environments of older people: A description of the psychosocial processes lining person to place. Journal of Gerontology, 44(2), S45-S53.

Sánchez-González, D. (2009). Contexto ambiental y experiencia espacial de envejecer en el lugar: el caso de Granada. Papeles de Población, 15(60), 175-213. Recuperado de http://www.redalyc.org/articulo.oa?id=11211 340008

Sánchez-González, D. (2015). Ambiente físico-social y envejecimiento de la población desde la gerontología ambiental y la geografía. Implicaciones socioespaciales en América Latina. Revista de Geografía Norte Grande, 60, pp. 97-114. Recuperado de https://scielo.conicyt.cl/scielo. php? script $=$ sci_arttext\&pid=S0718-34022015000100006

Sánchez-González, D. y Cortés, M. (2016). Espacios públicos atractivos en el envejecimiento activo y saludable. El caso del mercado de Terán, Aguascalientes (México). Revista de Estudios Sociales, 57, 52-67. Recuperado de http://www.redalyc.org/articulo.oa?id=81546458005

Satariano, W. A. (2006). Epidemiology of aging: An ecological approach. Boston: Jones and Bartlett Publishers.

Smith, A. E. (2009). Ageing in urban neighbourhoods. Place attachment and social exclusion. Bristol: The Policy Press-University of Bristol.

Soja, E. (2010). Seeking spatial justice. Minneapolis: University of Minnesota Press. 
Sokolec, J. (2016). The meaning of "place" to older adults. Clinical Social Work Journal, 44(2), 160-169. Recuperado de https://link.springer.com/ article/10.1007/s10615-015-0545-2

Ståhl, A. y Berntman, M. (2007). Falls in the outdoor environment among older persons. A tool to predict accessibility. En Proceedings of the 11th International Conference on Mobility and Transport for Elderly and Disabled Persons. Montreal: TRANSED.

Stevens-Ratchford, R. y Diaz, T. (2003). Promoting successful aging through occupation. An examination of engagement in life: A look at aging in place, occupation and successful aging. Activities, Adaptation \& Aging, 27(3-4), 19-37.

Thordardottir, B., Chiatti, C., Ekstam, L. y Malmgren Fänge, A. (2015). Heterogeneity of characteristics among housing adaptation clients in Sweden. Relationship to participation and self-rated health. International Journal of Environmental Research and Public Health, 13(1), 1-12. Recuperado de https://www.ncbi.nlm.nih.gov/pmc/articles/PMC4730482/ Tomasini, S. L. V. (2005). Envelhecimento e planejamento do ambiente construido: em busca de um enfoque interdisciplinar. Revista Brasileira de Ciencias do Envelhecimento Humano, 2(1), 76-88. Recuperado de http://seer.upf.br/index.php/rbceh/article/view/22/15

Vega, L. (2014). Ciudad, envejecimiento-vejez y educación: elementos para develar un conflicto entre la ciudad concebida y la ciudad practicada. Sophia, 10(1), 50-63. Recuperado de http://www.redalyc.org/pdf/4137/ 413734078005.pdf

Wahl, H.-W. y Gitlin, L. N. (2007). Environmental gerontology. En Encyclopedia of Gerontology. Oxford: Economic Press.

Wahl, H.-W. y Weisman, G. D. (2003). Environmental gerontology at the beginning of the new millennium: Reflections on its historical, empirical, and theoretical development. The Gerontologist, 43(5), 616-627. Recuperado de https://academic.oup.com/gerontologist/article/43/5/ $616 / 633791$

Wild, K., Wiles, J. L. y Allen, R. E. (2013). Resilience: Thoughts on the value of the concept for critical gerontology. Ageing and Society, 33(1), 137-158.

Wiles, J. L., Leibing, A., Guberman, N., Reeve, J. y Allen, R. E. (2011). The meaning of "ageing in place" to older people. The Gerontologist, 52(3), 357-366. Recuperado de https://academic.oup.com/gerontologist/article/ $52 / 3 / 357 / 580905$

Wilson, K. B. (2007). Historical evolution of assisted living in the United States, 1979 to the present. The Gerontologist, 47(SIII), 8-22. Recupe- 
rado de https://academic.oup.com/gerontologist/article/47/suppl_1/ $8 / 614189$

Zamorano, C., de Alba, M., Capron, G. y González, S. (2012). Ser viejo en una metrópoli segregada: adultos mayores en la Ciudad de México. Nueva Antropología , 25(76), 83-102. Recuperado de http://www.redalyc. org/articulo.oa?id $=15924294005$

\section{Acerca de los autores}

María Trinidad García-Valdez es candidata a doctora en Desarrollo Regional por el Centro de Investigación en Alimentación y Desarrollo A.C. Cuenta con una maestría en Administración por el Instituto Tecnológico de Monterrey, un diplomado en investigación en Ciencias Administrativas por la Universidad Autónoma de Ciudad Juárez y ha realizado una estancia de investigación en la Universidad Autónoma de Madrid. Posee experiencia profesional en el área administrativa, contable y financiera en el sector privado, así como entrenamiento en temas de análisis estadístico, liderazgo, servicio al cliente, trabajo en equipo, economía y proyectos de inversión.

Diego Sánchez-González es doctor en Geografía y maestro en Gerontología Social por la Universidad de Granada. Es profesor del Departamento de Geografía de la Universidad Autónoma de Madrid (España). Pertenece al Sistema Nacional de Investigadores, nivel II (Conacyt, México). Ha visitado y enseñado en universidades españolas y mexicanas, participando en la dirección de tesis de posgrado; también ha fungido como responsable y evaluador de proyectos de investigación a nivel internacional. Asimismo, participa como miembro de comités editoriales de revistas indexadas internacionales. Sus líneas de investigación son: geografía del envejecimiento, gerontología ambiental, envejecimiento activo, ciudades amigables con las personas mayores, envejecimiento vulnerable y cambio climático, y turismo y personas mayores. ORCID: http://orcid.org/0000-0002-2337-8917

Entre sus últimas publicaciones destacan:

Rivera Herrera, N. L., Ledezma Elizondo, M. T. y Sánchez-González, D. (coords.) (2017). Espacios públicos inclusivos. Monterrey, México: Universidad Autónoma de Nuevo León / Universidad Autónoma de Madrid.

Sánchez-González, D. y Rodríguez Rodríguez, V. (coords.) (2016). Environmental gerontology in Europe and Latin America. Policies and perspectives on environment and aging. Springer (Serie International Perspectives on Aging, 13). 
Sánchez-González, D., Adame Rivera, L. M. y Rodríguez-Rodríguez, V. (2018). Paisaje natural y envejecimiento saludable en el lugar: el caso del Parque Nacional Cumbres de Monterrey (México). Boletín de la Asociación de Geógrafos Españoles, 76, 20-51.

Rosario Román-Pérez es doctora en Ciencias Sociales por El Colegio de Michoacán. Actualmente es profesora-investigadora del Centro de Investigación en Alimentación y Desarrollo, A.C. (CIAD). Es integrante del Sistema Nacional de Investigadores. Sus líneas de investigación son: género, salud y educación. ORCID: http://orcid.org/0000-0002-2337-8917

Entre sus publicaciones recientes destacan:

Román-Pérez, R. y Cárdenas González, V. (coords.) (2016). La violencia en México. Problemas, estrategias y modelos de intervención desde las ciencias sociales. México: CIAD / UAM-I, AM Editores.

Román-Pérez, R., Domínguez, E., Saucedo, S. y Tánori, J. (2016). Validación de un instrumento sobre cultura de género en instituciones de educación, salud e investigación en el noroeste de México. La Ventana, 44, 83-108. García Puebla, M. D., Román, R., Alemán H. y Valenzuela, B. (2015). Identidades en transición: el caso de las personas adultas mayores. En: S. Garay Villegas, M. C. Arroyo Rueda y J. E. Bracamontes Grajeda (coords.), Vejez, familia y bienestar. Dimensiones micro y macrosociales del envejecimiento y la vejez. México: Universidad Autónoma de Nuevo León.

Fecha de recepción: 18 de octubre de 2017.

Fecha de aceptación: 6 de febrero de 2018. 